\title{
Wear of Media during Ultra-fine Grinding ${ }^{\dagger}$
}

\author{
Kazuo Suzuki, Yoshitaka Kuwahara \\ and Toshio Ishizuka \\ Government Industrial Research Institute, Nagoya \\ Agency of Industrial Science and Technology, M.I.T.I. *
}

\begin{abstract}
Contamination during fine grinding is an important problem for a raw powder of newly developed materials. Wear behaviors of grinding media, which were made of steel, alumina and zirconia, were examined during fine grinding using a vibration ball mill. Ball wear was nearly proportional to a specific surface area of ground products. It was supposed that the wear in wet grinding is more than in dry grinding, because wet grinding is more effective for fine grinding. Wear was effected by the materials of the grinding media. Ball wear, which was made of zirconia, was the least of three kinds of balls, that were used. Linear wear was not as much as ball wear.
\end{abstract}

\section{Introduction}

Powder is rarely a final product, but is handled, much more frequently, as an intermediate product, as is the case with starting material for the newly developed materials. It is increasingly required that powders be finer as the starting material for the new materials, and its particle and surface characteristics, chemical composition, crystalline phase have been attracting more attention. Grinding is a common procedure to reduce particle size in the production of preparation of powders, which are increasingly required to be finer.

In grinding operation, a physical and mechanical energy supplies to the powder. It decreases the particle size and, at the same time, produces new surface. However, it wears down the grinding media because of the frequently applied contact force. The grinding procedure is invariably accompanied by this type of problem and causes the powder to be contaminated with fine solids from the grinding components. The extent of contamination tends to increase as the size of the grinding particle decreases.

* 1-1 Hirate-cho, Kita-ku, Nagoya 462 Japan TEL. 052-911-2111

$\dagger$ This report was originally printed in J. Soc. Powder Technology, Japan, 26, $411-416$ (1989) in Japanese, before being translated into English with the permission of the editorial committee of the Soc. Powder Technology, Japan.
This problem is more pronounced in the fine grinding operation.

It is accepted that the wear mechanisms fall into four general categories ${ }^{1)}$; (1) adhesive, (2) abrasion, (3) corrosive wear and (4) surface fatigue. Each mechanism rarely appears by itself but in combination with the others. Furthermore, the extent of the actual wear varies greatly and depends on many factors, such as the atmosphere and operating conditions to which it is exposed.

Abrasive is one type of powder. Abrasion is distinguished from wear, but these may be regarded as the same phenomenon in that an abrasive causes volume reduction (or dimensional change) in materials it contacts. An abrasive consists of hard particles of which efficiently grind something or remove foreign matter therefrom. Extensive wear may result if the ground particles are harder than the grinding medium.

Wear caused by ball milling was extensively studied in 1940's, from the respective of controlling operations ${ }^{2)}$. As a result, the mechanisms of the ball wear are considered to fall into the three general categories ${ }^{3)}$ : The first and the second are based on the concepts that wear rate is proportional to the ball surface area $\left(d^{2}\right)$ and the ball mass $\left(d^{3}\right)$, respectively, and the third is intermediate between the two, assuming that the wear rate is proportional to $d^{n}(2<n<3)$, where $d$ stands for the ball diame- 
witer. These studies were carried out with steel balls and mainly directed at controlling the grinding speed to understand the relationships between ball size reduction and the ball makeup methods. The wear phenomena results were analyzed in which abrasive wear played a predominant role yet contamination of the powder was not discussed.

This study investigated the wear of media both for dry and wet grinding using a vibration ball mill, with emphasis on powder contamination. Silica sand served as the sample powder and three different materials were used for the grinding medium.

\section{Experimental Procedure and Sample}

The grinding apparatus used in this study was a high-vibration, high amplitude mill (nanoVMILL with two containers, Chuo Kakoki Co., Ltd.), whose amplitude (total amplitude) (from 4 to $19 \mathrm{~mm}$ and whose frequency varied up to 3400 c.p.m. by an inverter. In this study, the amplitude and frequency were set up at $10 \mathrm{~mm}$ and 1200 c.p.m., respectively, and its vibration strength was 8.06 relative to the acceleration of gravity. The grinding media (balls) were of HD alumina $(3,5$ and $10 \mathrm{~mm}$ in diameter, Nippon Kagaku Togyo Co., Ltd.), YTZ zirconia (3, 5 and $10 \mathrm{~mm}$ in diameter, Tosoh Co., Ltd. Nippon Kagaku Togyo Co., Ltd.), and carbon steel $\left(3 \mathrm{~mm}\left(1 / 8^{\prime \prime}\right)\right.$ and $5 \mathrm{~mm}\left(3 / 16^{\prime \prime}\right)$ in diame- ter, Tsubakimoto Seiki Co., Ltd.). The charged quantity of each grinding medium was set up at $J=0.7$. Two types of mill containers were used; one was lined with HD alumina (Nippon Kagaku Togyo Co., Ltd.) for the ceramic balls, and the other was of steel for the steel balls. These were $170 \mathrm{~mm} \phi \times 200 \mathrm{~mm}(4.5 \ell)$ and $200 \mathrm{~mm} \phi \times 195.6 \mathrm{~mm}(6.1 \mathrm{\ell})$, respectively, in size.

The sample powder was of silica sand $\left(\mathrm{SiO}_{2}\right.$ : 99.85\%, \#200, Kyoritsu Yougyo Genryo Co., Ltd.), ground by both the dry and wet processes, the latter was performed in the presence of water. The charged quantity of the sample powder was set at $U=1.14$. For the wet grinding, the concentration and charged quantity of slurry were set at $40 \mathrm{wt} \%$ and $114 \%$, respectively. These conditions are summarized in Table 1.

The grinding time was as much as 100 hours for wet grinding and 50 hours for dry grinding. A small quantity was sampled at given time intervals, to monitor the wear rate and specific surface area changing with time. The same quantity of water was made up in wet grinding.

The wear rate was followed in two ways; chemical analysis of sampled powder and weight loss of the grinding balls. The ball was weighted before and after the test; it was thoroughly washed with pure water to recover the ground product and dried before being measured after the test. The chemical analysis

Table 1 Experimental conditions

\begin{tabular}{|c|c|c|c|}
\hline \multirow{2}{*}{ Mill container } & \multirow{2}{*}{ Ball } & \multicolumn{2}{|c|}{ Powder } \\
\hline & & Dry grinding & Wet grinding \\
\hline $\begin{array}{l}\text { Alumina lining pot } \\
\text { Internal volume } \\
: 4.5 \ell\end{array}$ & $\begin{array}{l}\text { Alumina or zirconia } \\
(3,5,10 \mathrm{~mm}) \\
\text { Weight of ball } \\
: 6.20 \mathrm{~kg} \\
\text { (Alumina) } \\
: 10.50 \mathrm{~kg} \\
(\text { Zirconia }) \\
(J=0.70)\end{array}$ & $\begin{array}{l}\text { Weight of powder } \\
\quad: 1.70 \mathrm{~kg} \\
(U=1.14)\end{array}$ & $\begin{array}{l}\text { Weight of powder } \\
\quad: 0.75 \mathrm{~kg} \\
\text { Weight of water } \\
\quad: 1.12 \mathrm{~kg} \\
\text { Concent. of slurry: } \\
\quad: 40 \mathrm{wt} \% \\
\quad(U=1.14 \text { in slurry })\end{array}$ \\
\hline $\begin{array}{l}\text { Steel pot } \\
\text { Internal volume } \\
\quad: 6.1 \ell\end{array}$ & $\begin{array}{r}\text { Steel }(3,5 \mathrm{~mm}) \\
\text { Weight of ball } \\
: 19.70 \mathrm{~kg} \\
(J=0.70)\end{array}$ & $\begin{array}{l}\text { Weight of powder } \\
\qquad \begin{array}{l}: 2.30 \mathrm{~kg} \\
(U=1.14)\end{array}\end{array}$ & $\begin{array}{l}\text { Weight of powder } \\
\quad: 1.00 \mathrm{~kg} \\
\text { Weight of water } \\
\quad: 1.50 \mathrm{~kg} \\
\text { Concent. of slurry } \\
\quad: 40 \mathrm{wt} \% \\
(U=1.14 \text { in slurry) }\end{array}$ \\
\hline
\end{tabular}


was performed by means of an ICP luminescence analyzer (ICPA-100S, Nippon Jarrell Ash Co., Ltd.), with $\mathrm{Al}$ as the trace element for the alumina balls, $\mathrm{Zr}$ for the zirconia balls ( $\mathrm{Al}$ was used simultaneously for the liner), and $\mathrm{Fe}$ for the steel balls. Figure 1 shows sample preparation procedure for ICP analysis. The analytical conditions were: ICP output: $1.4 \mathrm{~kW}$, carrier gas rate: $0.52 \mathrm{l} / \mathrm{min}$, plasma gas rate: $14 \mathrm{l} / \mathrm{min}$, auxiliary gas rate: $0.52 \mathrm{\ell} / \mathrm{min}$, measurement wavelength: AlI $396.15 \mathrm{~nm}$ for the alumina balls. AlI $394.40 \mathrm{~nm}$ for alumina liner, FeII $238.20 \mathrm{~nm}$ and ZrII $348.82 \mathrm{~nm}$. (a) Sample ground by steel ball

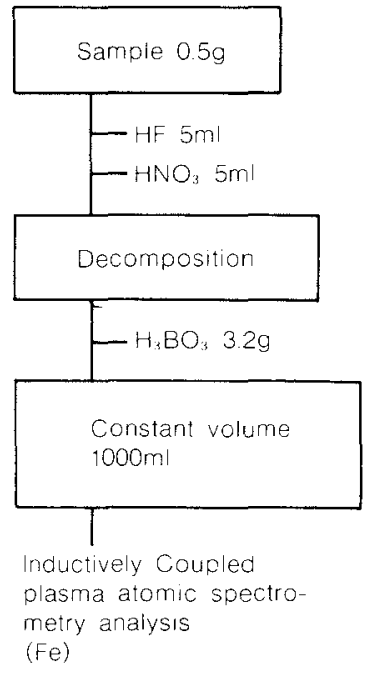

(Fe) (b) Sample ground by alumina or zirconia ball

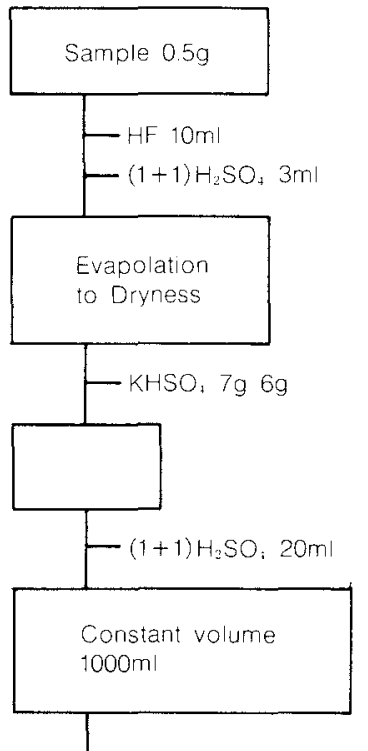

Inductively Coupled plasma atomic spectrometry analysis (A1 and/or.Zr)
Fig. 1 Analytical procedures of $\mathrm{Fe}, \mathrm{Al}$ and $\mathrm{Zr}$ in ground $\mathrm{SiO}_{2}$ powder

The specific surface area was determined by the $\mathrm{N}_{2}$ gas absorption type BET methods (Carlo Elba, Sorptomatic 1800).

\section{Results and Discussions}

Figure 2 shows the parity plot, where the wear debris fraction in $\mathrm{SiO}_{2}$ powder determined by the chemical analysis is plotted against that determined by the weight balance of the ball on a log-log graph. The solid line represents the $1: 1$ relationship between them. It is only natural that the values for the zirconia ball case are the same, because only the $\mathrm{ZrO}_{2}$

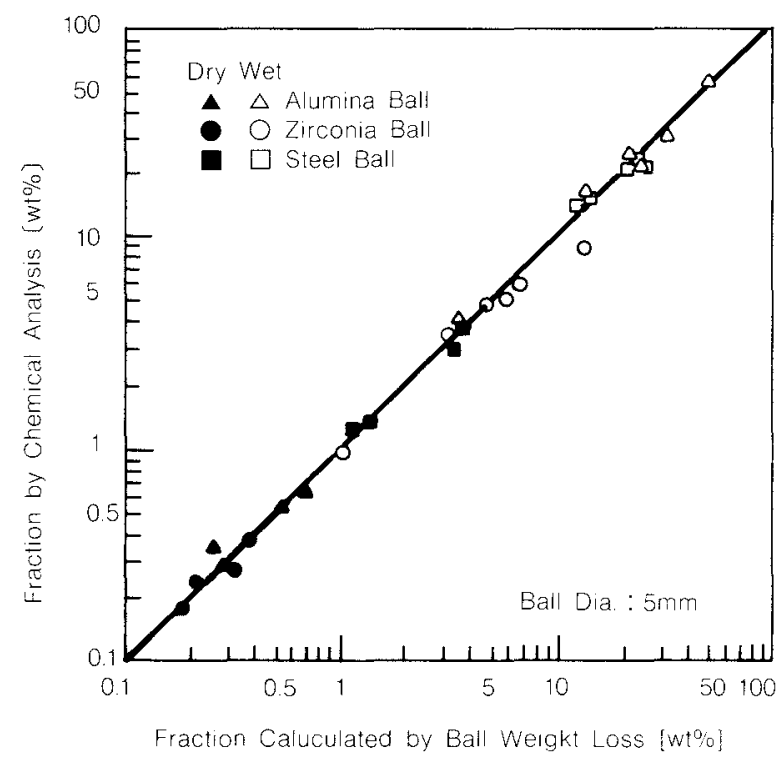

Fig. 2 Comparison of measured wear debris fraction in $\mathrm{SiO}_{2}$ powder between two methods

concentration resulting from the balls was chemically analyzed (concentration of alumina as the wall material was analyzed separately). For the steel and alumina ball case, however, the chemically analyzed value should be higher, with respect to the wear debris from the walls. Nevertheless, they coincided well with each other, suggesting that the quantities of debris from the walls were very small and, at the same time, the chemical analysis results were reliable.

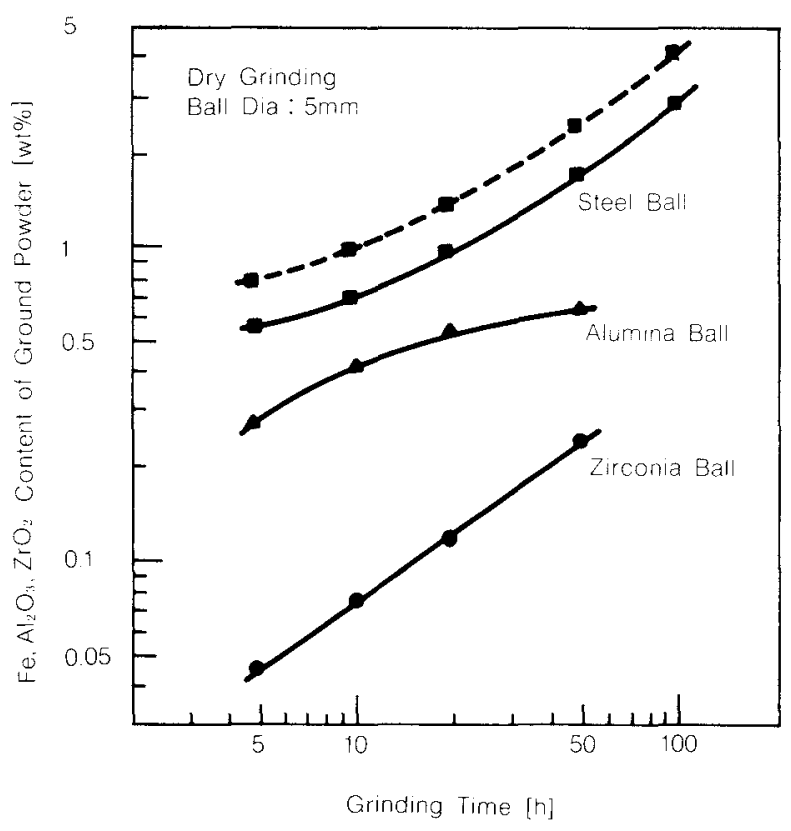

Fig. 3 Ball wear behaviors under dry grinding 
Figure 3 shows $\mathrm{Fe}, \mathrm{Al}_{2} \mathrm{O}_{3}$ and $\mathrm{ZrO}_{2}$ concentrations in the ground powder over time for the dry grinding case. The broken line presents the $\mathrm{Fe}_{2} \mathrm{O}_{3}$ concentration in the powder ground by the steel balls. It serves as a comparison for the later presented wet grinding results, which discuss $\mathrm{Fe}_{2} \mathrm{O}_{3}$ concentration. The wear of the balls, is presented by Fe quantity, which, however, is in all likelihood oxidized into $\mathrm{Fe}_{2} \mathrm{O}_{3}$ when present in the ground powder. Of the 5 mm-diameter balls tested, the steel ball produced the largest quantity of the wear debris, followed by the alumina and then the zirconia ball. Wear is often associated with relative hardness of the materials involved. In general, hardness of steel, alumina and zirconia are $\mathrm{Hv} 400$, 2000 and 1300, respectively, while that of silica sand is $\mathrm{Hv} 800$. Thus, steel satisfies the condition that causes abrasive wear by the ground silica sand powder. It was observed, however, that the wear debris quantity was not proportional to the grinding time, meaning that the wear, even if abrasive wear predominated, was affected by ground powder characteristics (extent of size reduction during the process), in addition to the ball size (surface area or mass), and that the wear could not be explained by abrasive wear alone, because the vibration ball mill used in this study used impact force as grinding mechanism. The relationship between

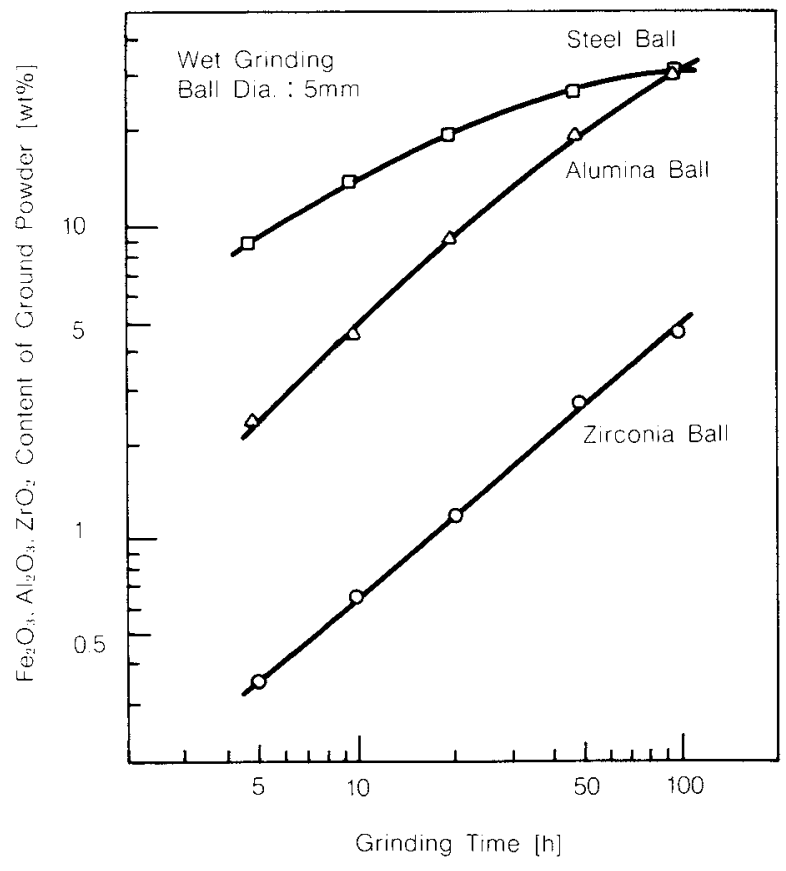

Fig. 4 Ball wear behaviors under wet grinding wear rate and hardness was reversed for the results with the alumina and zirconia balls.

Figure 4 presents the wet grinding results, where the balls had the same diameter as those used for the dry grinding and where the debris from the steel balls was represented by iron oxide $\left(\mathrm{Fe}_{2} \mathrm{O}_{3}\right)$, estimated from the elementary $\mathrm{Fe}$ concentration in the powder, because $\mathrm{Fe}$ was considered to be oxidized. As for the wet grinding, the zirconia balls demonstrated the smallest wear rate. Comparing the results shown in Fig. 4 with those in Fig. 3 revealed that the debris produced by the wet grinding was approximately one order of magnitude greater than that by the dry grinding.

As described earlier, grinding with the zirconia balls was performed in the alumina lined container. Analysis of $\mathrm{Zr}$ and $\mathrm{Al}$ concentrations in the ground powder, therefore, gave the

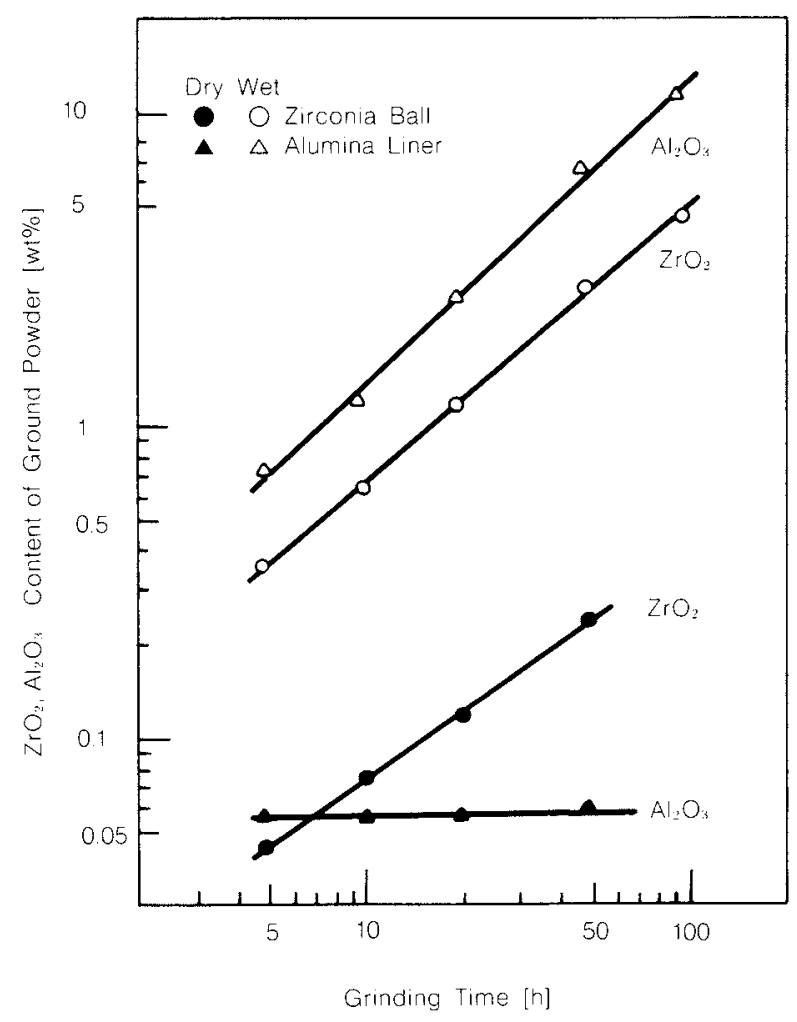

Fig. 5 Wear behaviors of alumina vessel and zirconia ball

quantities of the balls and the lining worn during the grinding process. Figure 5 shows the analytical results, indicating that the lining was worn quite differently during the dry and wet grinding process. During wet grinding impact of the balls were directly applied continuously to 
the alumina walls, because the powder was dispersed in the grinding fluids, with the result that quantity of worn alumina increased with grinding time. During dry grinding, on the other hand, the extent of wear of the alumina walls was limited, except during the first grinding stage before the walls were coated with a layer of ground powder. An increase in the worn quantity with time was not observed. It is possible that the ground powder coating the walls greatly absorbed the impact of the balls and protected the walls. The balls were also coated with the powder, but the layer was probably thin as it repeatedly exfoliated from, and reattached to, the balls were continuously worn, though their wear rate was approximately one order of magnitude lower than that observed during wet grinding.

The wear of alumina and zirconia balls and the alumina walls during wet grinding was further investigated by the other test. It was conducted for 50 hours under almost the same conditions, except that no powder was used. The same diameter $(5 \mathrm{~mm})$ balls, in $1.5 \ell$ of water were used to measured the wear of balls. During the test, the slurry samples containing the worn powder were collected after 5 and 20 hours. The quantity of worn balls during 50 hours was measured by balance. The quantity of liner worn during the 50 hours grinding was estimated from the measured slurry density and the quantity of worn balls. The quantities of the balls and liner worn by 5 and 20 hours were also estimated.

Table 2 Results of wear test without powder in water

\begin{tabular}{|c|c|c|c|}
\hline Materials. & Ball wear & Liner wear & Total wear \\
\hline Alumina & & & \\
\hline $5 \mathrm{~h}$ & $36.8(48.5)$ & $16.9(5.0)$ & $53.7 \quad(53.5)$ \\
\hline $20 \mathrm{~h}$ & $147.0(155.5)$ & $24.6(16.0)$ & $171.6(171.5)$ \\
\hline $50 \mathrm{~h}$ & 367.5 & 37.8 & 404.3 \\
\hline Zirconia & & & \\
\hline $5 \mathrm{~h}$ & $(2.7)$ & $8.5 \quad(8.2)$ & $10.9(10.9)$ \\
\hline $20 \mathrm{~h}$ & $(9.6)$ & $29.5(29.6)$ & $39.1 \quad(39.2)$ \\
\hline $50 \mathrm{~h}$ & 24.1 & 74.1 & 98.2 \\
\hline
\end{tabular}

The results are shown in Table 2, where the slurry concentrations estimates are based on the assumption that the balls were worn at a constant rate. The estimated results in paren- theses are based on the assumption that the ratio of wear quantity between balls and liner was constant. Densities of the alumina ball and liner are described later. The specific surface areas measured 26.1 ( 5 hours) to 45.1 (50 hours) $\mathrm{m}^{2} / \mathrm{g}$ with the powder from the worn alumina balls, and 12.9 (5 hours) to 34.0 (50 hours) $\mathrm{m}^{2} / \mathrm{g}$ with the worn zirconia balls (including the worn alumina powder from the liner). This indicates that they were fairly fine. It was therefore considered that all closed pores in the sintered body surfaced in the worn powders and that their densities were slightly higher than those provided by manufacturer (alumina: 3.60 , zirconia: 6.00 ). The manufacturer's values were used in the calculation however.

As shown in Table 2, the wear rate of the liner was fairly low, approximately $10 \%$ the anticipated results as shown in Fig. 2. This and the results shown in Fig. 2 (except for the zirconia ball results), showed that the liner was less worn than the ball, when similar materials were used as the grinding media. When the balls of dissimilar materials (zirconia balls and alumina liner in this case) were used, on the other hand, the alumina liner was worn significantly more, and great care must be taken in such case. It should be noted, however, that the wear rate in the presence of ground powder (Alumina: $193.4 \mathrm{~g}$, Zirconia: $21.6 \mathrm{~g}$ (Alumina: $56.6 \mathrm{~g}$ )) was lower in each case, as revealed by the 50 hours grinding tests. It is possible that the ground powder dampened the balls' impact on the liner walls, even during the wet grinding.

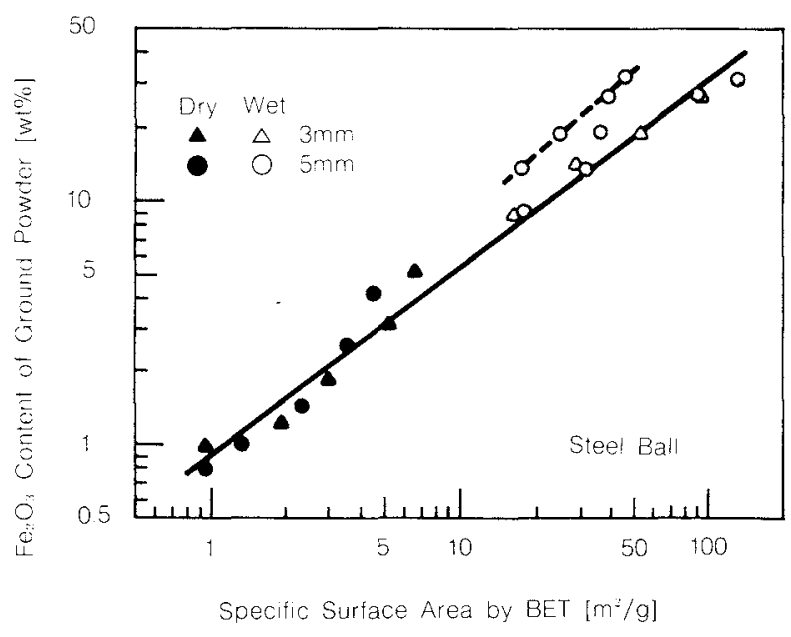

Fig. 6 Relation between specific surface area and contamination of powder ground by steel ball 
W The densities of the alumina ball and liner, provided by manufacturer were 3.60 and 3.50 , respectively, which are considerably lower than well-densified alumina at 3.98 . The alumina ball consisted of sintered $\mathrm{Al}_{2} \mathrm{O}_{3}(91 \%), \mathrm{SiO}_{2}$ $(7 \%)$ and unknowns (2\%), and may have contained $10 \%$ or more of glassy and spinel phases in the grain boundaries and a number of pores. Both the glassy and spinel phases were low in hardness and strength, and the presence of large quantities of the grain boundaries diminished the inherent characteristics of alumina therefore aggravating the wear of the alumina products. The zirconia ball had a density of 6.00 , which was very close to the theoretical density of 6.05 . Zirconia, though not very strong, its toughness helps reduce its wear.

Figure 6 through 8 show the relationships between the specific surface area of ground powder and contents of $\mathrm{Fe}_{2} \mathrm{O}_{3}, \mathrm{Al}_{2} \mathrm{O}_{3}$ and $\mathrm{ZrO}_{2}$, respectively, where the specific surface area is the value of the ground powder containing the wear debris. Figure 6 shows the steel ball results. The broken line represents the wet grinding results (marked with o's) with the $5 \mathrm{~mm}$-diameter balls, where the samples were treated with an acid to remove the $\mathrm{Fe}_{2} \mathrm{O}_{3}$ wear debris. The relationship with the wear debriscontaining powder is represented by a straight line on a $\log -\log$ plot, indicating that the wear debris content was closely related to the specific surface of the ground powder and that the

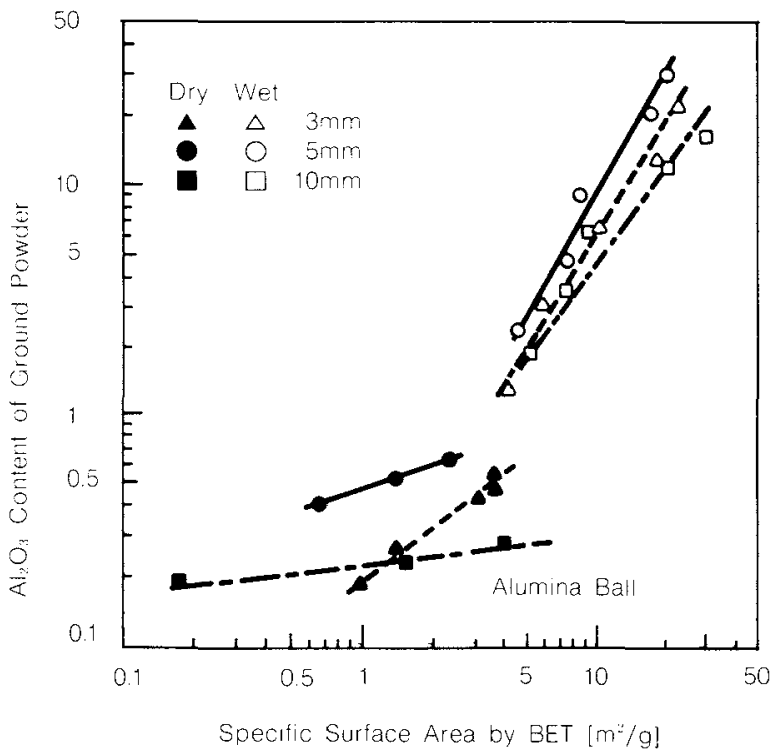

Fig. 7 Relation between specific surface area and contamination of powder ground by alumina ball

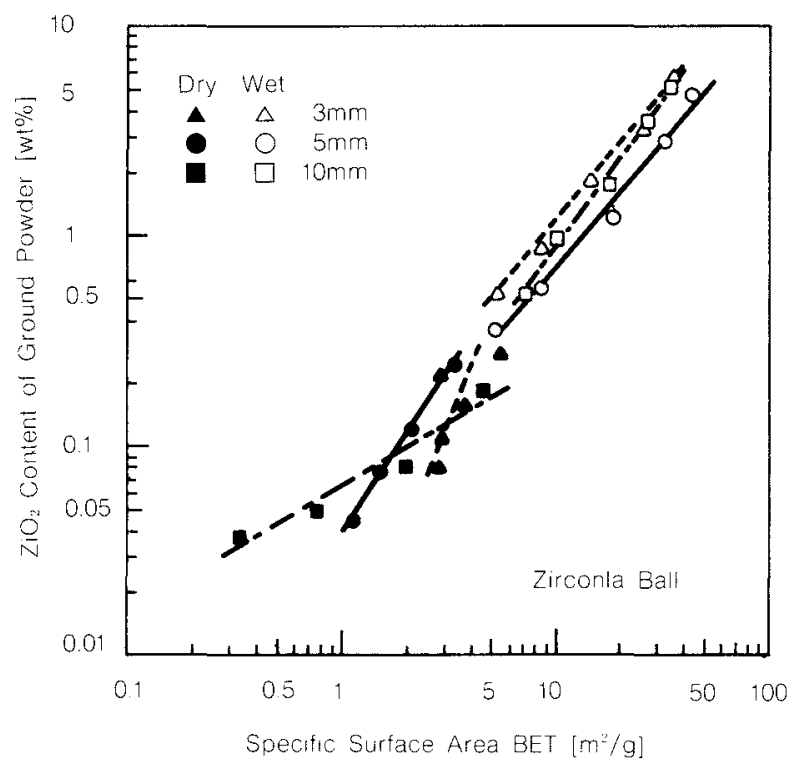

Fig. 8 Relation between specific surface area and contamination of powder ground by zirconia ball

grinding methods whether dry or wet had no effect on the relationship, though they produced very different amounts of wear debris.

The acid-treated powder had a significantly reduced specific surface area, which means that the wear debris of steel was somewhat finer. Figure 7 and 8 present the results with the alumina and zirconia balls, respectively. Unlike the case with the steel balls, the dry grinding produced smaller quantities of wear debris, and caused the content to be less sensitive to the specific surface of the powder than the wet grinding. In any case, no significant increase in specific surface area is expected from dry grinding.

The effects of the ball size were hidden by the effects of the mechanical properties of the different materials. During wet grinding, the wear debris quantity tended to increase as specific surface area of ground powder increased. It is therefore believed that the ground powder is somewhat contaminated with the wear debris when making a very fine powder.

\section{Conclusions}

Three types of grinding media were used both for dry and wet grinding in a vibration ball mill, to investigate extent of wear debris contamination in the ground powder.

(1) The extent of contamination was essentially proportional to the specific surface area 
of the ground powder in any case of what grinding medium material was used. Wet grinding, which grinds powder at a higher rate than dry grinding, will cause the ground powder more contamination.

(2) Of the ball types used in the tests, the zirconia ball produced the least amount of debris, followed by the alumina ball and then the steel ball.

(3) The liner was generally less worn than the balls. However, care must be taken if the liner and the ball are of dissimilar materials.

(4) Wear of the grinding medium in the impact mechanism seems to be more affected by the materials' strength than the ball size (mass or surface area).

\section{Acknowledgements}

The authors thank Mr. Fumisaku Maruyama, president of Chuo Kakoki Co., Ltd., for his support in this study, and Messrs. Kazumitsu Murakami and Tatsuyoshi Ito for their cooperation.

\section{Nomenclature}

$J:$ ball filling of mill (ratio of volume of balls plus space between balls to volume of mill)
$U$ : powder fraction (ratio of volume of powder particles plus space between particles to volume of space between balls)

\section{References}

1) Hashimoto K.: "Funtaimamou no Taisaku", p.2, Nikkan Kougyou Shinbunsya (1981).

2) Prentice T.K.: J. Chem. Met. Min. Soc., South Africa, Jan. 99 (1943), White H. A.: Trans. AIME, 153, 116 (1943), Garms W. I. and J. L. Stevene: Trans. ALME, 169, 133 (1943), Norman T.E. and C. M. Leob: Mining Tech., AIME Tech. Pub. No. 2319, 1 (1948), Norman T.E. and C. M. Leob: Trans. AIME, 183, 330 (1943), Norquist D. E. and J. E. Moeller: Trans. AIME, 187, 721 (1950), Rose H. E. and R.M.E. Sullivan, "A Treatise on the Internal Mechanics of Ball, Tube and Rod Mills", Constable London, 1957, Lorenzetti J. J.: Proc. 3rd Symp. on Grinding, Armco Chile, S.A.M.I. Vina del Mar 284 (1980), Davis E.W. Trans. AIME, 61, 250 (1919), Longmore E. L.: Trans. Inst. Min. Met., 46, 569 (1937), Crocker B. S.: J. Chem. Met. Min. Soc. South Africa, Feb. 133 (1944), Bond F. C.: Trans. AIME, 153, 373 (1943), Bond F. C.: J. Chem. Met. Min. Soc. South Africa, Jan. 131 (1944), Vermeulen L. A. and D. D. Howat: Mintek Report, M201, 1 (1985).

3) Menacho J. and F. Concha: Powder Tech., 47, 87 (1986). 